\title{
Proof of Space Transactions: A Novel Blockchain Protocol for Secure Authentication of Satellite Transactions
}

\author{
Aboul Ella Hassanien ( $\square$ aboitcairo@gmail.com ) \\ Cairo University \\ Mohamed Torky \\ Higher Institute of Computer Science and Information Systems \\ Essam Goda \\ Arab Academy for Science, Technology and Maritime Transport \\ Vaclav Snasel \\ Technical University of Ostrava \\ Tarek Gaber \\ University of Salford
}

\section{Research Article}

Keywords: PoST, Ethereum GAZ, science exploration, weather forecasting, imagery, communications, transparent, verifiable, secure manner

Posted Date: August 23rd, 2021

DOI: https://doi.org/10.21203/rs.3.rs-820584/v1

License: (c) (1) This work is licensed under a Creative Commons Attribution 4.0 International License. Read Full License 


\title{
Proof of Space Transactions: A Novel Blockchain Protocol for Secure Authentication of Satellite Transactions
}

\author{
Mohamed Torky ${ }^{1,+}$, Essam Goda ${ }^{2,+}$, Aboul Ella Hassanein ${ }^{3,+,}$, Vaclav Snasel $^{4}$, and Tarek \\ Gaber $^{5,6,+}$
}

${ }^{1}$ Higher Institute of Computer Science and Information Systems, Culture \& Science City, Computer Science, 6 October City, postcode 12573, Egypt

${ }^{2}$ College of Computing and Information Technology (CCIT), Arab Academy for Science, Technology, and Maritime Transport (AASTMT) , Computer Science, Cairo, postcode 12611, Egypt

${ }^{3}$ Faculty of Computer and Artificial Intelligence, Cairo University, Information Technology, Cairo, Postcode 12631, Egypt

${ }^{4}$ VSB-Technical University of Ostrava, Czech Republic

${ }^{5}$ School of Science, Engineering, and Environment, University of Salford, Manchester, M5 4WT,UK

${ }^{6}$ Faculty of Computers and Informatics, Suez Canal University, Ismailia, Postcode 41522, Egypt

+Scientific Research Group in Egypt (SRGE)

*Corresponding.aboitcairo@cu.edu.eg)

\begin{abstract}
Blockchain technology can play a vital role in the space industry and satellite communication. This disruptive technology can build decentralized and secure protocols for processing and manipulating space transactions in the form of space digital tokens (SDTs). Tokenizing space transactions in the form of SDTs will enable various blockchain-based applications in the space industry. Moreover, blockchain protocols based on smart contracts can be utilized to authenticate many space transactions and P2P communications in a transparent, verifiable, and secure manner. This paper proposes a new blockchain-based solution for managing and securing satellite transactions using a novel concept called SDT. SDT is then used to develop a new blockchain protocol called proof of space transactions (PoST), which is then used in proposing a new blockchain-based protocol for authenticating satellite transactions. The proposed PoST protocol is implemented and simulated using the Ethereum blockchain. Five metrics are used to evaluate the protocol's performance: Ethereum GAZ, read latency, transaction latency, read throughput, and transaction throughput. The performance evaluation results proved the efficiency and reliability of PoST in managing and securing satellite transactions.
\end{abstract}

\section{Introduction}

According to the Space Report 2020 Q2 analysis, the global space economy reached $\$ 423.8$ Billion in 2019, which represents a $2.2 \%$ increase from 2018 (estimated at $\$ 414.75$ billion) ${ }^{1}$. Satellite communications technology provides two types of space activities: governmental and commercial. Governmental activities include civil applications (such as science exploration and weather forecasting) and military applications (such as imagery and communications). Commercial activities include launching, communications, and satellite imagery. It is very difficult to imagine modern life without satellite-based services, such as digital maps, navigation, and mobile communication. Such services are based on various types of space technologies and satellite transactions ${ }^{2}$. The design of new generations of satellite systems has become dependent on different various types of emergent technologies, such as artificial intelligence $(\mathrm{AI})^{3},{ }^{4},{ }^{5}$, the Internet of Things (IoT) ${ }^{6},{ }^{7},{ }^{8}$, edge computing $9,{ }^{9},{ }^{11}$, and quantum computing ${ }^{12},{ }^{13},{ }^{14},{ }^{15}$.

Blockchain technology has rapidly become a major technology that can be used to solve many challenges in the global space industry ${ }^{16},{ }^{17},{ }^{18}$. Within the global space industry, blockchain can create many opportunities, such as satellite-as-a-service business models, space supply chain management, and even satellite payloads construction. Moreover, blockchain is expected to cause a major transformation of the next generation of financial transactions. Transferring the Bitcoin blockchain via satellite will make a quantum leap in global financial transactions and grant a robust alternative to terrestrial networks. The blockstream satellite network ${ }^{19}$ represents a practical application of utilizing blockchain-based satellites for processing and broadcasting Bitcoin to the entire planet without the need for the internet. For instance, in 2017, a technical error by Google briefly caused more than half of Japan to lose connection to the internet. While internet access was returned within the hour, the Japanese 
faced slow connection speeds, which directly affected financial transactions, and online trading was halted. In this scenario, broadcasting the Bitcoin blockchain via satellite would have ensured that financial transactions were not halted. Moreover, blockchain remained in sync with the rest of the globe and was thus unaffected by the internet disconnect ${ }^{20}$.

Additionally, the distinguishing characteristics of blockchain technology, such as robustness, trust, security, transparency, decentralized connections, and time stamping transactions, make it a key technology for managing and securing space communications, whether among different satellites or satellites and ground stations, because it cannot be hacked or centrally controlled. These characteristics make blockchain a qualified technology for providing various services in the space industry ${ }^{20}$, such as the following:

- Enhancing the satellite value chain: Blockchain, as a smart contract, can be used to create transparency, trust, and efficiency in the satellite value chain. For example, smart contract-based applications can be developed to launch and operate satellites, access transparent information for insurance purposes, and monitor space operations. Moreover, satellites can also be basic sources of space transactional data for upgrading blocks and verifying the integrity and origin of these data patterns.

- Enabling cloud services in space: Leveraging both blockchain and AI can enable cloud transformation and processing in space. Blockchain over satellites removes the dependence on terrestrial networks for storage, broadcasting, or processing space data, thereby eliminating significant vulnerabilities for a data breach or distortion. This can be achieved based on cloud computation while processing space data at rest or in motion, from satellite to satellite or from an end-user to a satellite and vice versa.

- Tracking the satellite supply chain: Blockchain can be established as a tracking system to ensure and verify data during each phase of satellite procurement, design, testing, and launch. Blockchain can also be used to monitor a satellite's motion, share space data with all stakeholders, and enforce rules of any updates made to the satellite system that require the consensus of the team. In the future, the blockchain-satellite system will depend on cloud constellations for managing data centers in orbit, where companies can upload their data and bypass ground networks; this approach will help governments and companies obtain information from different sources and orbits in space ${ }^{20}$.

- Designing open-source satellites: Large space companies have begun to develop blockchain-based open-source satellite networks to provide many services to end-users on the ground and enable them to directly access satellite services. The Singapore-based Space Chain ${ }^{21}$ began to build the world's first open-source blockchain-based satellite network. Space Chains permits end -users to develop and run decentralized applications by accessing open-source satellites in space.End-users need to understand only the functionality of smart contracts without the need for more technical details of how the space industry works.

\subsection{Problem Statement and Research Questions}

Most global space agencies have begun to rely on the launch of constellations (or swarms) of satellites that work together to accomplish a specific space mission instead of relying on a single satellite ${ }^{22}$. Unlike a single satellite, a constellation can grant permanent global coverage at any time. Therefore, at least one satellite is available to respond to ground instructions at all times. Many types of satellite constellations have been launched for various missions, such as navigational satellite constellations (e.g., global positioning systems (GPSs)), communications satellite constellations (e.g., dish network and iridium (AIS, ADS-B)), and Earth observation satellite constellations (e.g., A-trains). A satellite constellation is vulnerable to some cyber-attacks that aim to disrupt one satellite or all satellites of the constellation, such as hacking, spoofing, interference, and jamming attacks $^{23}$. For instance, hacking the very small aperture terminal (VSAT) (VSAT is a small-sized ground station utilized to transmit/receive data, audio, and video signals over a satellite communication network, excluding television broadcasting) is the most common hacking technique that aims to access uplink and downlink data from/to VSAT devices ${ }^{24}$. Moreover, the failure to encrypt uplink/downlink data can expose satellites and ground stations to spoofing attacks ${ }^{25}$. In addition, jamming the control link of the uplink data may insert noise into ground stations' commands sent to a satellite or a constellation system. Furthermore, hijacking and control of satellite signals transmitted between two satellites or more or between satellites and ground stations represents another important security challenge in space communication. Therefore, authenticating space transactions, especially regarding satellite constellation networks, is an important issues to address in space communication security.

In response to these challenges, a novel blockchain protocol for managing and securing satellite transactions called proof of space transactions (PoST) is proposed. This protocol is based on a new concept called space digital tokens (SDTs). The main objective of the proposed PoST is to manage and authenticate satellite communications using blockchain. Three research questions must be answered in this study: 
1. How the proposed PoST can manage transactions between two satellites in the same constellation (or swarm).

2. How the proposed PoST can manage transactions between two or more satellites in different constellations.

3. What the reliability of the system is in securing communications against unauthenticated satellites that may attack the constellation with unauthorized connections

To achieve these objectives, the following assumptions are made:

- Satellites revolve around the earth within a swarm (or a constellation). Each swarm is managed by a country or a space agency.

- Communication between satellites within a specific swarm is conducted through a peer-to-peer network.

- The blockchain statues is shared with all satellites in the same swarm.

\subsection{Paper structure}

The rest of this paper is organized as follows: Section 2 presents a literature review. Section 3 discusses the concept, of SDTs). Section 4 discusses the proposed blockchain protocol,(PoST). Section 5 presents the simulation results. Section 6 discusses the results, and Section 7 presents conclusions and directions for future work.

\section{Literature Review}

A relatively small number of studies consider integrating blockchain technology into space industry applications. Lillian Clark et al. ${ }^{26}$ proposed a blockchain-based reputation system for developing a decentralized and secure system for satellite relay networks. The authors investigated and validated the network performance using average latency, computational complexity, and storage considerations for a variety of use cases. Feng and Hao Xu [16] studied the security problem for mobile satellite communication networks (MSNETs). The authors proposed a new security framework for securing mobile satellite communication networks based on reformulating satellite communication networks as delay-tolerance networks (DTNs). Blockchain was used with DTNs to 1) secure data transactions and 2) resist unexpected cyber-attacks that target mobile satellite networks by integrating blockchain with the practical satellite constellation management algorithm.

Songjie Wei et al. ${ }^{27}$ proposed a fast and efficient access verification protocol called the blockchain-based access verification protocol (BAVP), which integrates identity-based encryption and blockchain technology to authenticate LEO satellite constellations. BAVP represents a good alternative to traditional centralized authentication protocols that work for MEO/GEO satellite networks. The simulation results on the OPNET platform confirmed the reliability, effectiveness, and fast-switching efficiency of the proposed protocol. Additionally, Ronghua Xu et al. ${ }^{28}$ proposed a blockchain-based access control mechanism to address both access authorization issues and identity authentication in a distributed space network environment. The author implemented the proposed mechanism on both resource-constrained edge devices and more powerful devices and deployed it on a local private Ethereum blockchain network to evaluate the computational and timeliness performance.

Satellite data broadcasting is another problem that has been investigated in blockchain adoption in the space industry. Zhang YH and Liu XF ${ }^{29}$ introduced a new blockchain protocol that works based on satellite broadcasting communications instead of the traditional internet for data dissemination. Simulation results confirmed that the proposed technique achieved low communication cost and can improve the throughput of the blockchain system to 6,000,000 TPS with a 20 Gbps satellite bandwidth.

No approach in the literature, aims to manage and authenticate satellite communications using blockchain. Moreover, no work addresses the management of transactions between two satellites in the same constellation (or swarm) or transactions between two or more satellites in different constellations. These considerations will be addressed in this paper.

\section{Modeling Space Transactions as Space Digital Tokens (SDTs)}

In this section, a new concept, called SDTs, is proposed. SDTs are a way to tokenize space transactions as digital tokens that can be processed using a blockchain protocol to authenticate space transactions. SDTs can be broadcast within a swarm of satellite networks called a satellite constellations. Hence, blockchain can work in this scenario as an authenticator for all communication patterns that can occur within a specific satellite's constellation. SDTs can also be used to process sensing data between satellites and orbital debris; hence, blockchain can work in this scenario as a tracking system to detect expected space collisions between satellites and orbital debris.

Figure 1 depicts a model of SDTs using blockchain. and explains how space transactions can be modeled as SDTs and processed using a blockchain protocol. SDTs can represent a transaction exchanged from a satellite to a satellite within a P2P 
satellite network or sensing data between a satellite and orbital debris. The blockchain protocol is responsible for verifying the new space transactions to add a new valid block to the blockchain. All space stakeholders are then able to access the newly added blocks through the connected dashboard to the blockchain platform that manages a satellite constellation.

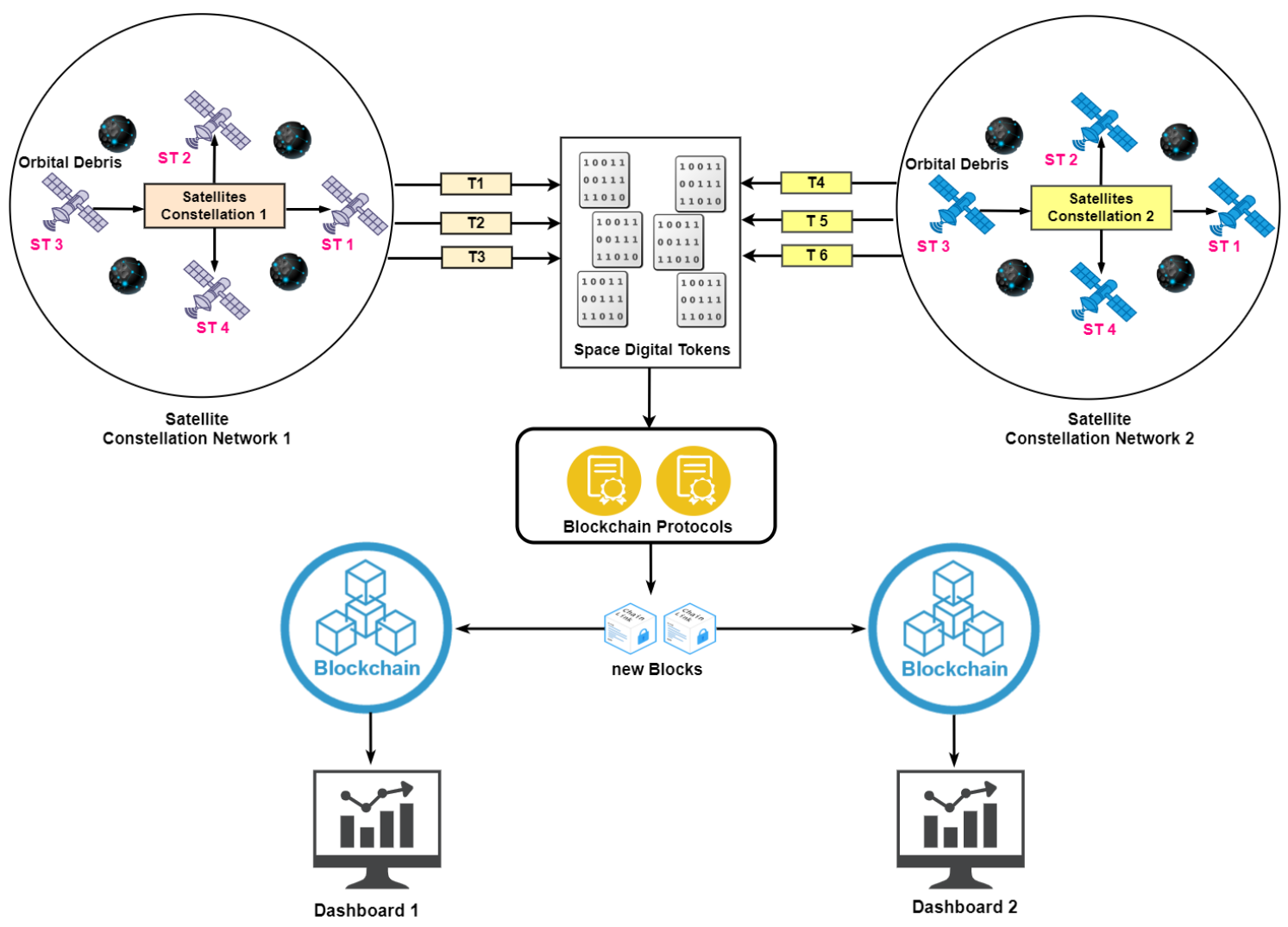

Figure 1. Modeling space digital tokens using blockchain

Algorithm 1 specifies how blockchain can be constructed based on processing SDT in space. Each space transaction has to be converted into an SDT). This new transaction (i.e., SDT) has to be verified using a blockchain protocol (or consensus) to confirm the validation of a specific transaction between two satellites in the same constellation. Additionally, the blockchain protocol is responsible for validating all transactions exchanged within a satellite constellation. If the handled SDT is valid, a new block is added to the blockchain. The new block contains all details of the new space transaction. This new information can be used by space stakeholders who are authorized to access the blockchain.

The main advantages of tokenizing space transactions as SDTs and processing them using the blockchain technology can be described as follows:

- Greater control over space transactions: Tokenizing space transactions into SDTs enhances the tracking, processing, verification, and securing of space behaviors triggered by space objects. Moreover, manipulating such transactions through a consortium blockchain system based on smart contracts supports self-verification and execution of satellite transactions. Hence, this approach leads to little dependency on ground stations. In addition, the intended space stakeholders will have greater control in terms of granting and revoking access to their satellites and spacecraft, as well as data sharing to and from a satellite network..

- Faster transactions and automated compliance: Compliance and fast response are major factors in space transaction management. Smart contracts based on blockchain could be beneficial in automating a variety of space transaction patterns, such as satellite-to-satellite (S2S), ground station-to-satellite (G2S), and vice versa, and user- to-satellite (U2S) and vice versa. The long time that a space message takes to reach ground stations can be eliminated using blockchain systems, where blockchain can manage uplink/downlink transactions between satellites and ground stations in real time.

- Security: Blockchain-enabled decentralization of space networks eliminates the risks involved in the traditional singlepoint-access model of space communications. Moreover, digitized space transactions on blockchain are cryptographically 


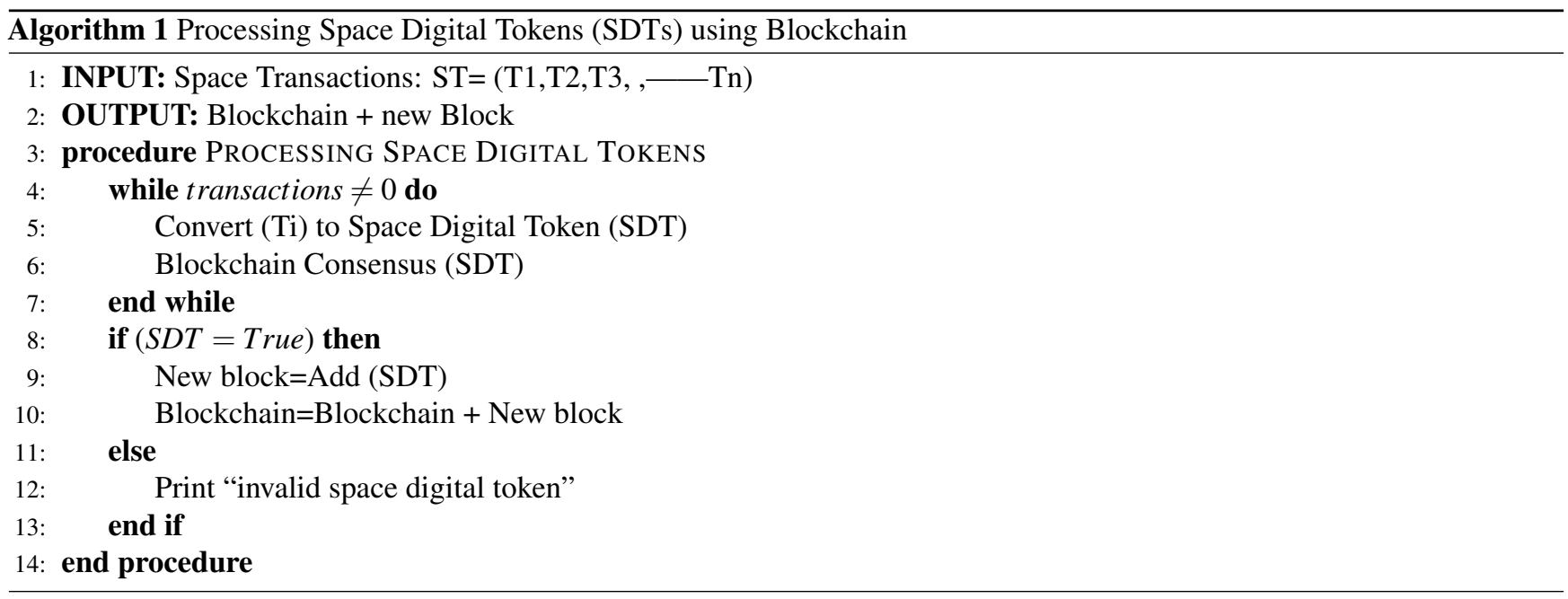

encrypted, making them tamper-proof. Additionally, blockchain enables space companies to track and monitor their satellites and spacecraft in a more controlled and flexible manner. Thus, blockchain will enable space agencies to detect any attacks on a satellite system that seeks to tamper with the satellite software system using space bots ${ }^{30}$.

\section{Proof of Space Transactions (PoST): A Blockchain Protocol for Authenticating Space Digital Tokens}

Proof of Space Transactions (PoST) is a novel blockchain protocol that can be utilized to verify SDTs within a satellite's constellation and to add a new block to the blockchain. The PoST methodology represents each satellite within a constellation as a private key and a piece of cryptographic evidence for a satellite's private key that is cryptographically attached to a specific SDT. When a new SDT is triggered between two satellites, the satellite that created the transaction shares the cryptographic evidence of this transaction with the rest of the satellite constellation to confirm the validity of the triggered SDT. In addition, the receiving satellite requests the nonce code of the last block of the blockchain. Once the nonce code is confirmed by the receiving satellite, a new block is added to the blockchain.

Figure 2 explains how the PoST protocol verifies and authenticates an SDT that has been exchanged between two satellites within the same constellation through eight steps. 


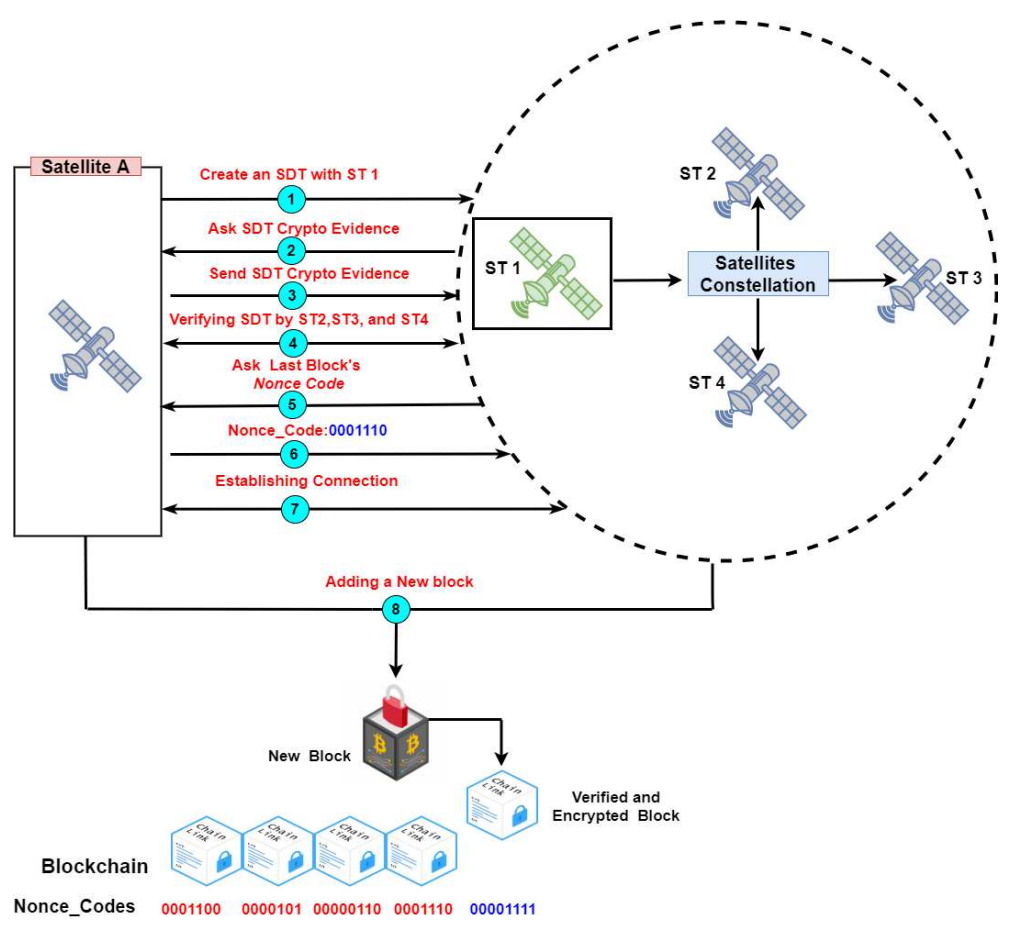

Figure 2. Proof of space transactions (PoST) protocol: Authenticating SDTs between two satellites within the same satellite constellation

Similarly, PoST can be used to verify SDTs between two satellites within different constellations (i.e., different satellite swarms) that work together for a specific purpose, as illustrated in Figure 3. In this authentication mode, each constellation is associated with a cryptographic code called constellation cryptographic evidence, which is used to establish secure transactions between two satellites within different constellations. Therefore, in this scenario, two verification processes are required: verifying the constellation cryptographic evidence between the two connected constellations (see step 5 in figure 3), and verifying the SDT cryptographic evidence between the two connected satellites within the two constellations (see step 6 in figure 3). Algorithm 2 specifies the PoST method for authenticating SDTs between two satellites within the same satellite constellation, while Algorithm 3 specifies the PoST method for authenticating SDTs between two satellites within different satellite constellations. 


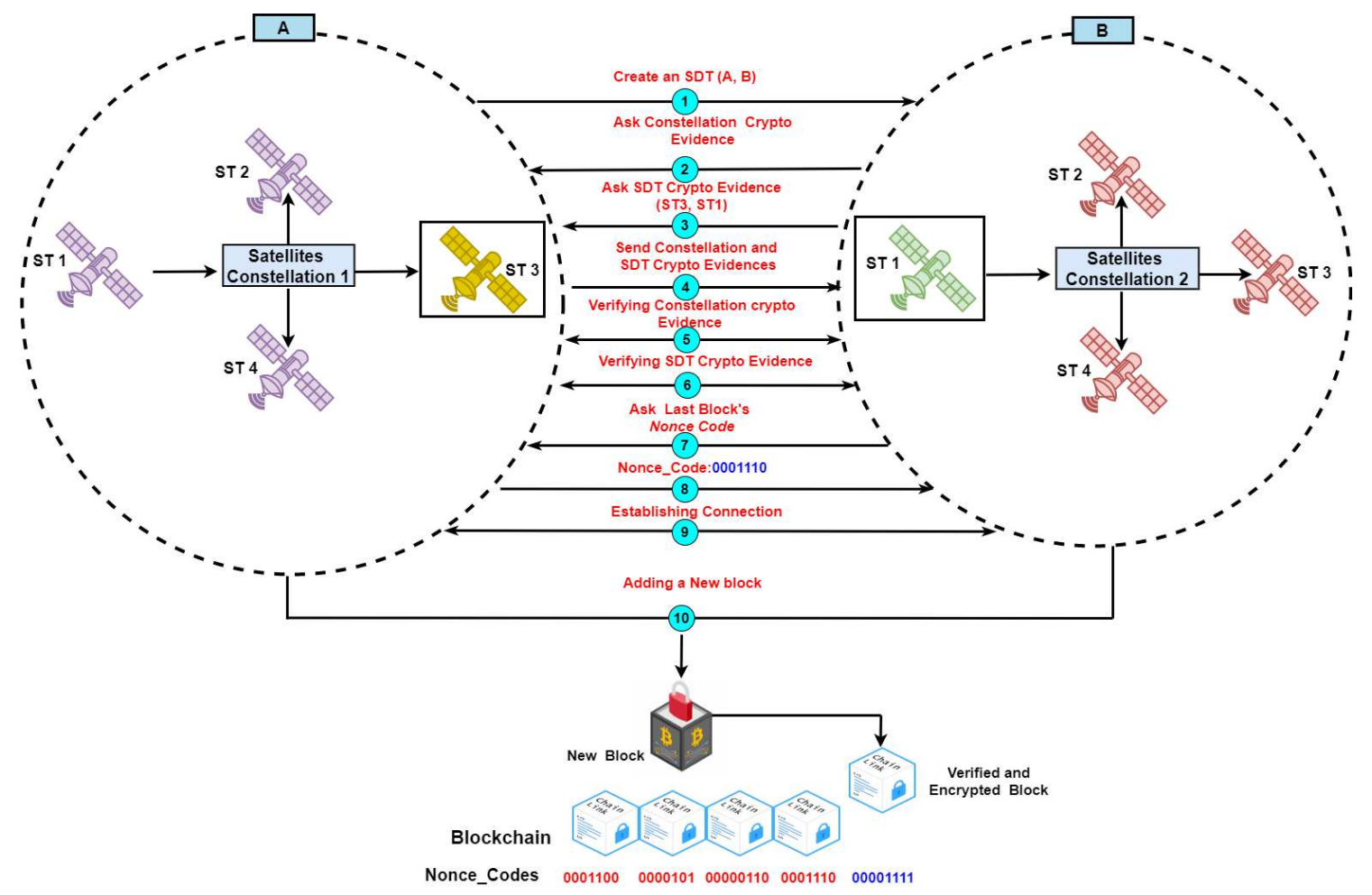

Figure 3. Proof of space transactions (PoST) protocol: Authenticating SDTs between two satellites within different satellite constellations.

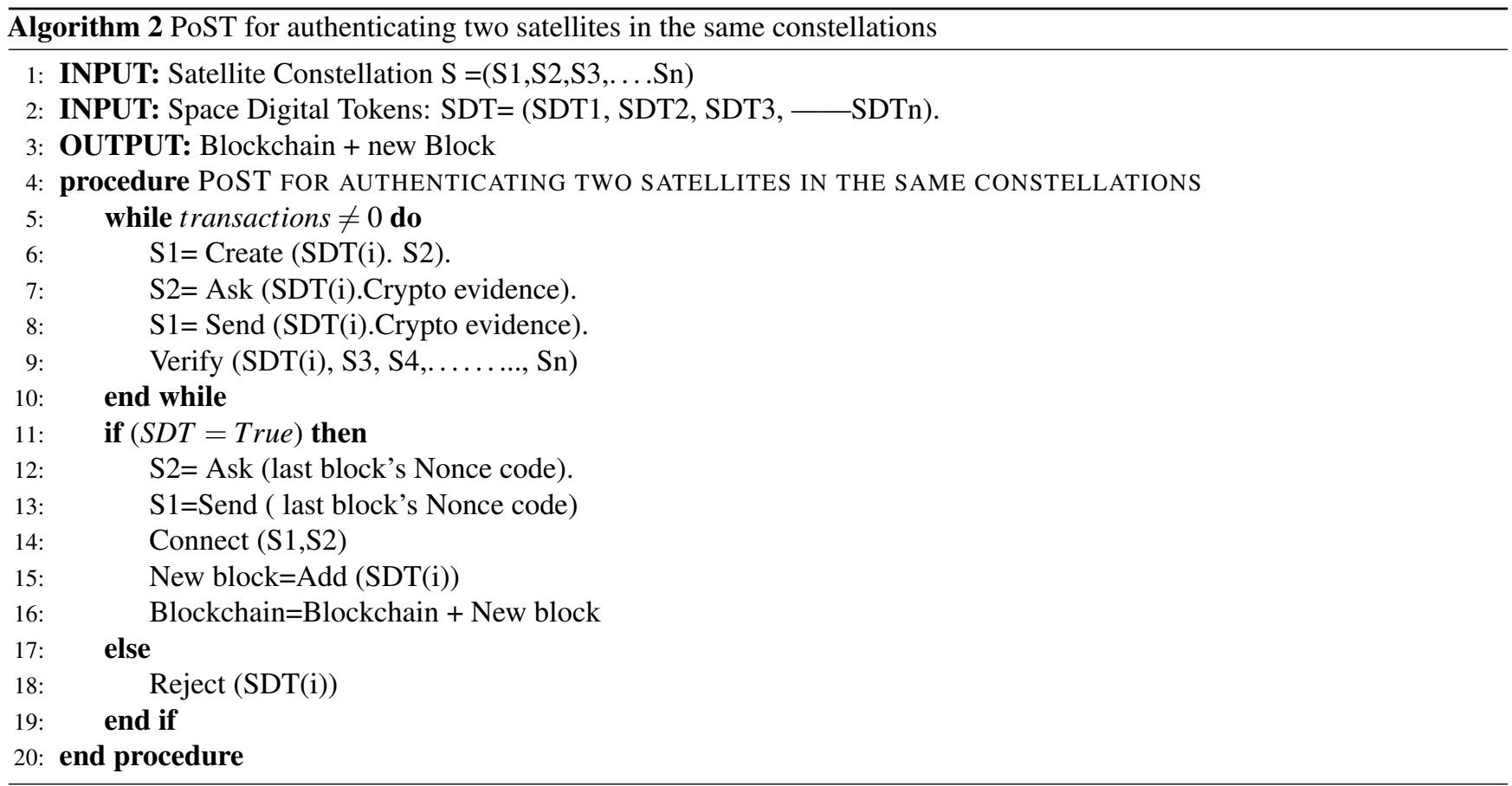




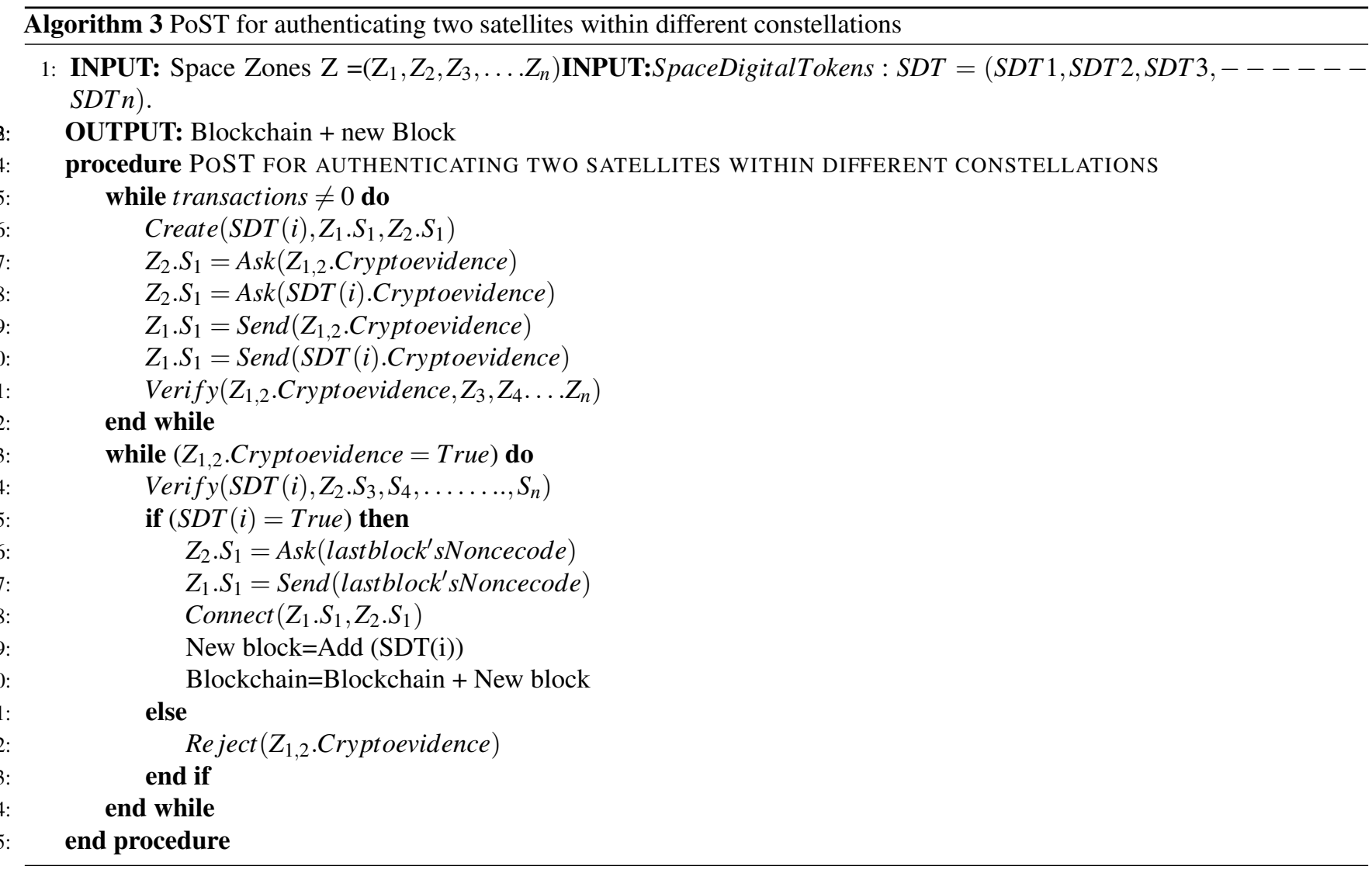

\section{Simulation Results}

To assess the efficiency of the proposed blockchain protocol, PoST, a dataset was created, and two simulation experiments were conducted.

\subsection{PoST implementation}

To implement the PoST protocol, we used the following software tools:

- Python: used to implement the authentication algorithms and integration between all system components.

- Ethereum: A blockchain platform used to simulate PoST functionality as a smart contract.

- Truffle: testing framework and asset pipeline for blockchain using the Ethereum virtual machine (EVM)

\subsection{Dataset Description}

The dataset consists of three swarms of satellites, A, B, and C. Each swarm consists of 10 satellites. Table 1 shows the satellite transactions that have been exchanged between swarms A, and B, where the size of the transaction data is 1049 bytes. Table 2 shows the satellite transactions that have been exchanged between swarms B, and C, where the size of the transaction data is 2049 bytes. Table 3 shows the satellite transactions that have been exchanged between swarms C, and D, where the size of transaction data is 3049 bytes. For each transaction data size (i.e., 1049, 2049, and 3049), the proposed protocol was simulated on seven samples of transactions, with 50,100,150, 200, 250,300, or 350 transactions. As there are no benchmark data suitable to evaluate PoST, the dataset was created using the Python random library, as depicted in Tables 1, 2, and 3. 


\begin{tabular}{|l|l|l|l|l|l|l|l|l|l|}
\hline A/A & S2 & S10 & S4 & S5 & S1 & S7 & S3 & S8 & SUM \\
\hline S1 & 7 & 10 & 8 & 6 & - & 6 & 4 & 9 & 50 \\
\hline S2 & - & 13 & 19 & 15 & 17 & 10 & 14 & 12 & 100 \\
\hline S7 & 20 & 24 & 18 & 19 & 25 & - & 21 & 23 & 150 \\
\hline S9 & 21 & 25 & 25 & 29 & 27 & 24 & 26 & 24 & 200 \\
\hline S5 & 38 & 32 & 30 & - & 33 & 39 & 36 & 42 & 250 \\
\hline S10 & 45 & - & 38 & 44 & 36 & 50 & 47 & 40 & 300 \\
\hline S6 & 39 & 46 & 40 & 47 & 50 & 43 & 48 & 37 & 350 \\
\hline
\end{tabular}

Table 1. Satellites transactions within Swarm A (the size of a transaction is 1049 bytes).

\begin{tabular}{|l|l|l|l|l|l|l|l|l|l|}
\hline A/B & S6 & S10 & S8 & S5 & S3 & S7 & S4 & S9 & SUM \\
\hline S1 & 7 & 4 & 8 & 6 & 7 & 5 & 8 & 5 & 50 \\
\hline S2 & 14 & 12 & 16 & 12 & 8 & 18 & 11 & 13 & 100 \\
\hline S3 & 18 & 23 & 16 & 25 & 14 & 17 & 21 & 16 & 150 \\
\hline S9 & 28 & 23 & 27 & 29 & 33 & 24 & 19 & 17 & 200 \\
\hline S5 & 29 & 38 & 33 & 17 & 29 & 36 & 33 & 35 & 250 \\
\hline S6 & 27 & 43 & 39 & 35 & 41 & 33 & 44 & 38 & 300 \\
\hline S10 & 48 & 33 & 41 & 50 & 40 & 51 & 43 & 44 & 350 \\
\hline
\end{tabular}

Table 2. Satellites transactions between Swarms A, and B (the size of a transaction is 2049 bytes).

\begin{tabular}{|l|l|l|l|l|l|l|l|l|l|}
\hline B/C & S10 & S6 & S4 & S1 & S7 & S5 & S3 & S8 & SUM \\
\hline S2 & 5 & 6 & 7 & 4 & 9 & 8 & 4 & 7 & 50 \\
\hline S6 & 13 & 12 & 9 & 17 & 15 & 9 & 14 & 11 & 100 \\
\hline S7 & 21 & 13 & 24 & 15 & 22 & 15 & 23 & 17 & 150 \\
\hline S9 & 26 & 17 & 21 & 28 & 30 & 29 & 24 & 25 & 200 \\
\hline S5 & 30 & 29 & 33 & 39 & 26 & 37 & 32 & 24 & 250 \\
\hline S10 & 34 & 38 & 49 & 33 & 36 & 39 & 40 & 31 & 300 \\
\hline S8 & 55 & 51 & 48 & 40 & 36 & 45 & 39 & 36 & 350 \\
\hline
\end{tabular}

Table 3. Satellites transactions between Swarms B, and C (the size of a transaction is 3049 bytes).

\subsection{Experimental Design}

Two main simulation experiments were designed to evaluate the performance of the PoST protocol. The purpose of the first experiment was to evaluate the blockchain performance in managing satellite transactions either within the same swarm (i.e., a constellation as specified in Algorithm 2) or between two different swarms, as specified in Algorithm 3. The purpose of the second experiment was to evaluate the reliability and authenticity of the proposed PoST

\subsubsection{Blockchain performance results}

Five metrics were used to evaluate blockchain performance: Ethereum GAZ, read latency, transaction latency, read throughput, and transaction throughput:

1. Ethereum $G A Z$ refers to the fee required to successfully execute a transaction on the Ethereum blockchain platform. GAZ is represented as small fractions of the cryptocurrency ether (ETH), commonly referred to as Gwei and sometimes also called nanoeth. GAZ is used to assign resources to the EVM) so that decentralized applications, such as smart contracts, can self-execute in a secured but decentralized manner. Hence, our study measured how computationally expensive a satellite transaction is or how much transaction processing is needed.

2. Read latency $(R L)$. Before we define read latency and how it is computed, it is important to differentiate between reading and transaction operations in the blockchain. The read operation refers to an internal mechanism that can be implicitly executed by a blockchain node to fetch the required data to verify specific transactions, but it does not update the blockchain status. On the other hand, a transaction is a state transition that updates data in the blockchain by adding a new block to the chain. Therefore, a transaction is explicitly executed by a blockchain node and verified 
using a blockchain protocol against a set of rules called a smart contract. If a transaction is valid, the blockchain system will commit the transaction and add a new block to the chain containing all details of this transaction. In our study, a transaction can be satellite communication or sensing of debris moving toward a constellation zone or satellite orbit. Therefore, the RL) is the time between when the read request is submitted by a satellite and when the response is received. Equation 1 formulates the RL calculation

$$
R L=\text { Response time }- \text { Submission time }
$$

3. Transaction latency $(T L)$ refers to a blockchain network-wide view of the amount of time taken for a transaction from creation to be available across the blockchain network. Equation 2 is used to compute the TL

$$
\text { TL=Confirmation time@networkthreshold-submission time }
$$

4. Read throughput $(R T)$ is a measure of how many read operations are executed in a specified time interval, expressed as reads per second (RPS). Equation 3 is used to calculate RT

$$
R T=\sum(\text { Reads Operations }) / \sum(\text { Times in seconds })
$$

5. Transaction throughput (TT) is the rate at which valid transactions are executed by the blockchain system in a defined time interval. This is not the rate at a single blockchain node, but across the entire blockchain network. This rate is expressed as transactions per second (TPS) at a specified network size. Equation 4 is used to calculate TT

$$
T T=\sum(\text { Commited Transactions }) / \sum(\text { Times in seconds })
$$

We designed and conducted three experiments to assess the blockchain performance:

- Experiment 1: This experiment investigates the impact of transaction size on the performance of the proposed system within a single swarm, where the size of all transactions is 1049 bytes. The experiment was applied to seven samples of satellite transactions (50T, 100T, 150T, 200T, 250T, 300T, and 350T). Figure 4 depicts the obtained results that clarify the impact of a transactional data size equal to 1049 bytes on RL, and TL after applying Algorithm 2 to the dataset depicted in Table 1.

- Experiment 2: This experiment investigates the impact of transaction size on the performance of the proposed system when transactions occur between two satellites within two swarms, where the size of all transactions is 2049 bytes. The experiment was applied to seven samples of satellite transactions (50T, 100T, 150T, 200T, 250T, 300T, and 350T). Figure 5 depicts the obtained results that clarify the impact of a transactional data size equal to 2049 bytes on RL, and TL after applying Algorithm 2 to the dataset depicted in Table 2.

- Experiment 3: This experiment investigates the impact of transaction size on the performance of the proposed system within two swarms, but in this scenario, we increased the size of all transactions to 3049 bytes. The experiment was also applied to seven samples of satellite transactions (50T, 100T, 150T, 200T, 250T, 300T, and 350T). Figure 6 depicts the obtained results that clarify the impact of a transactional data size equal to 3049 bytes on RL and TL after applying Algorithm 2 to the dataset depicted in Table 3.

Moreover, Figure 7 depicts the relationship between the transaction data size and Ethereum GAS. 


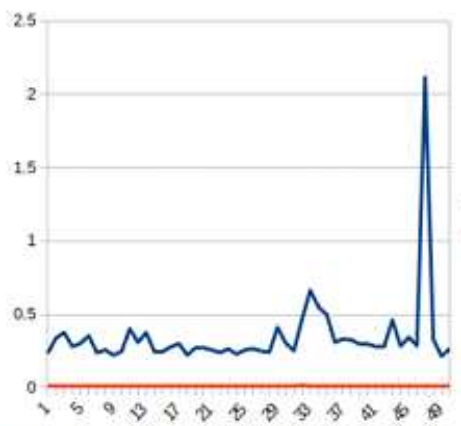

a) (1049 bytes, 50 transactions)
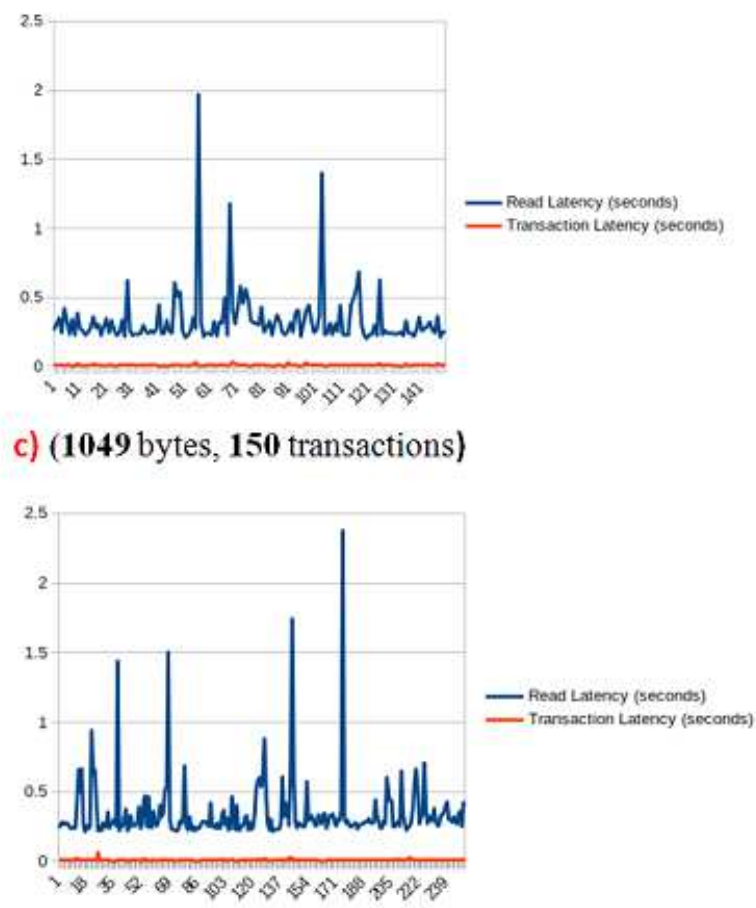

e) (1049 bytes, 250 transactions)

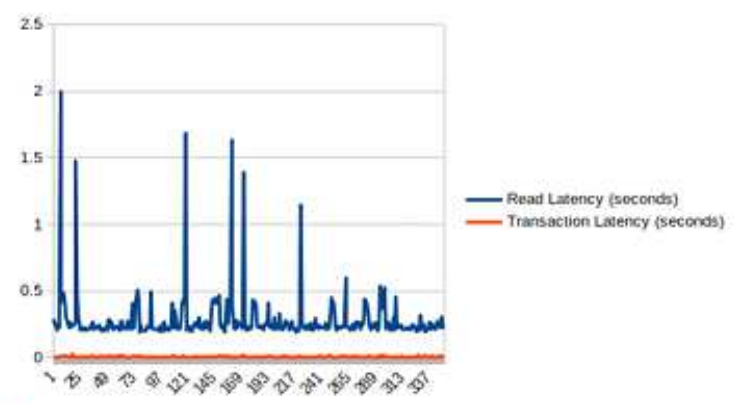

g) (1049 bytes, 350 transactions)

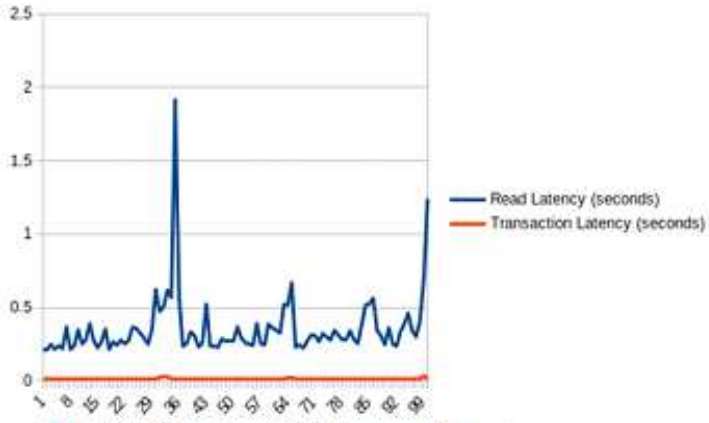

b) (1049 bytes, 100 transactions)

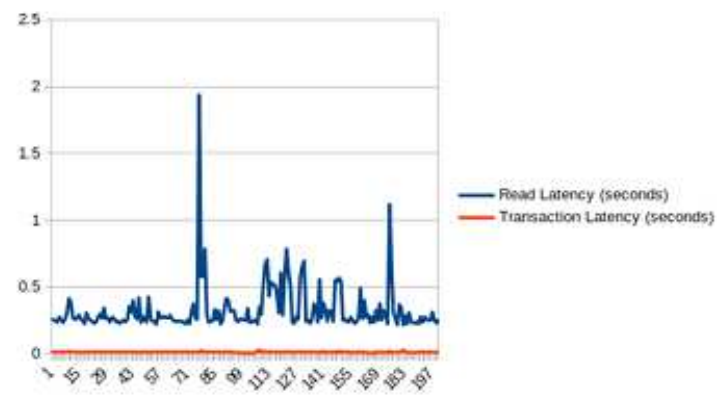

d) (1049 bytes, 200 transactions)

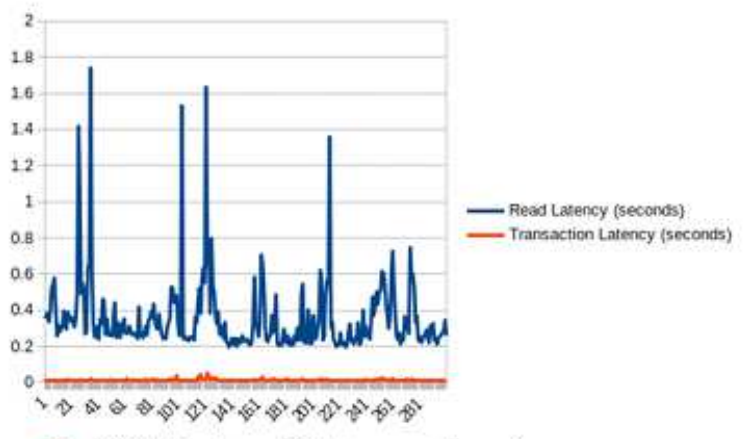

f) (1049 bytes, 300 transactions)

Figure 4. Impact of a transaction data size of 1049 bytes on RL, and TL for 50, 100,150,200,250,300, and 350 transactions. 


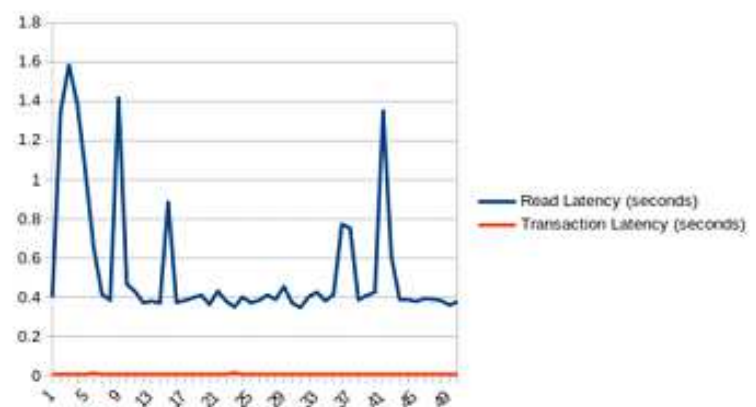

a) (2049 bytes, $\mathbf{5 0}$ transactions)
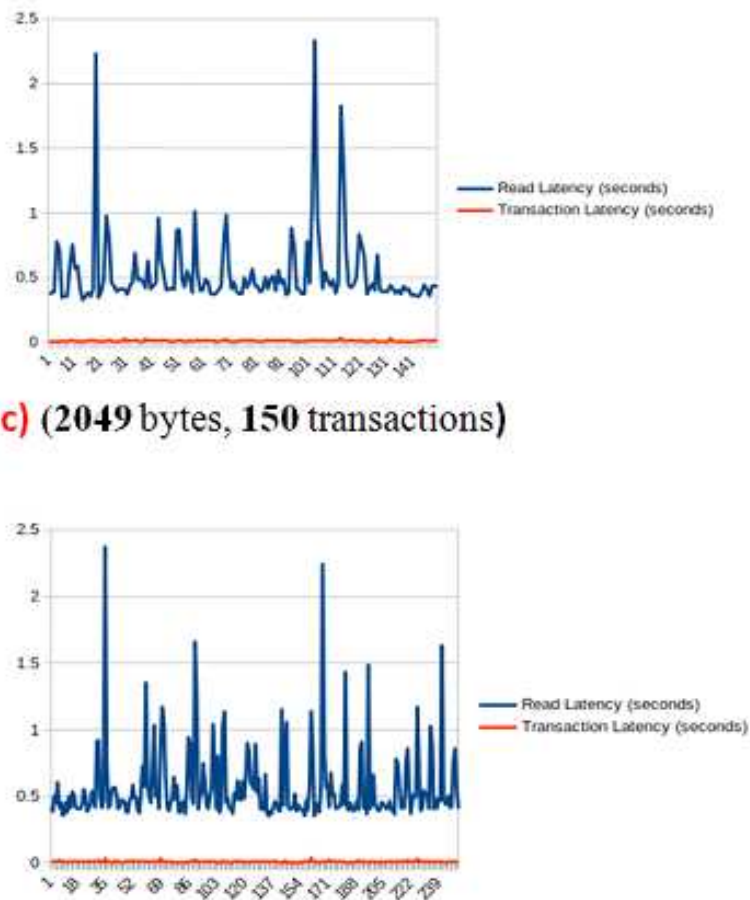

e) (2049 bytes, 250 transactions)

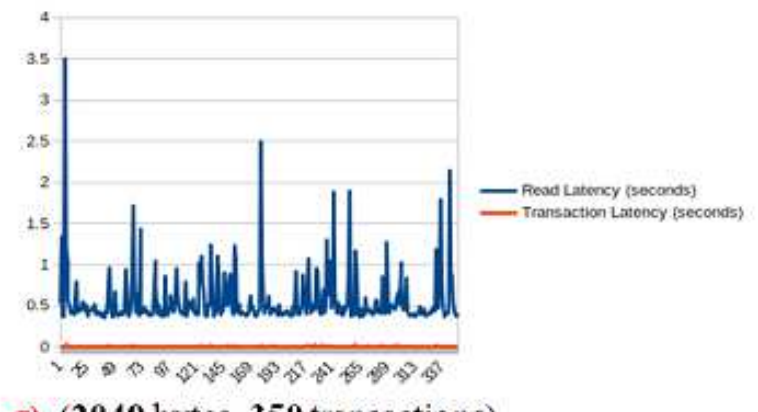

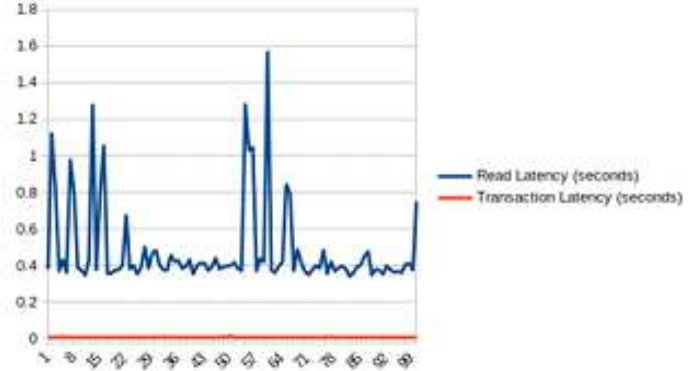

b) (2049 bytes, 100 transactions)

$\odot$

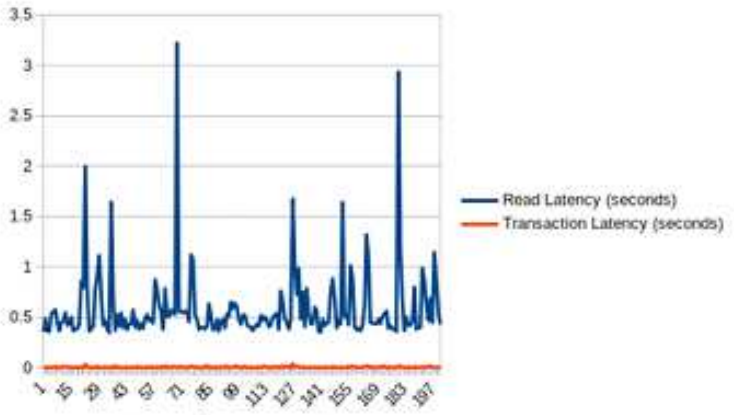

d) (2049 bytes, 200 transactions)

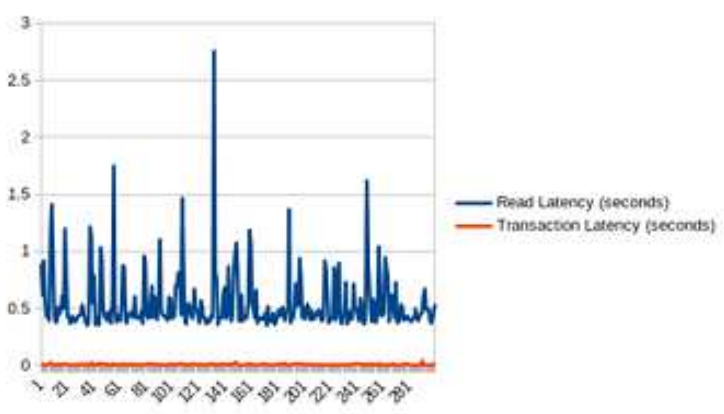

f) (2049 bytes, 300 transactions)

Figure 5. Impact of a transaction data size of 2049 bytes on RL, and TL for 50, 100,150,200,250,300, and 350 transactions. 

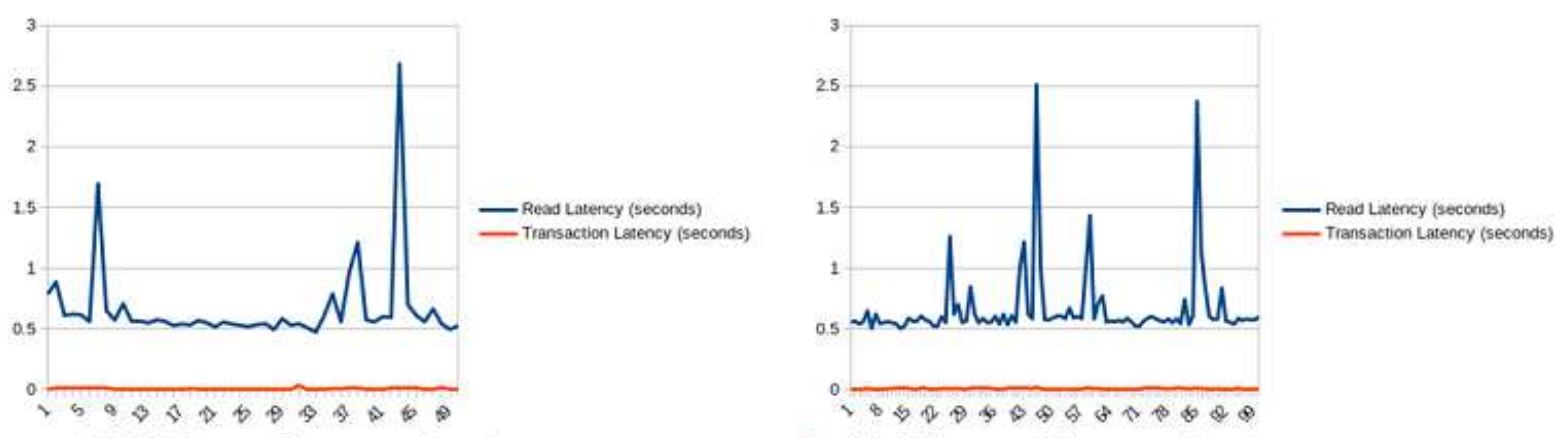

a) (3049 bytes, $\mathbf{5 0}$ transactions)

b) (3049 bytes, 100 transactions)
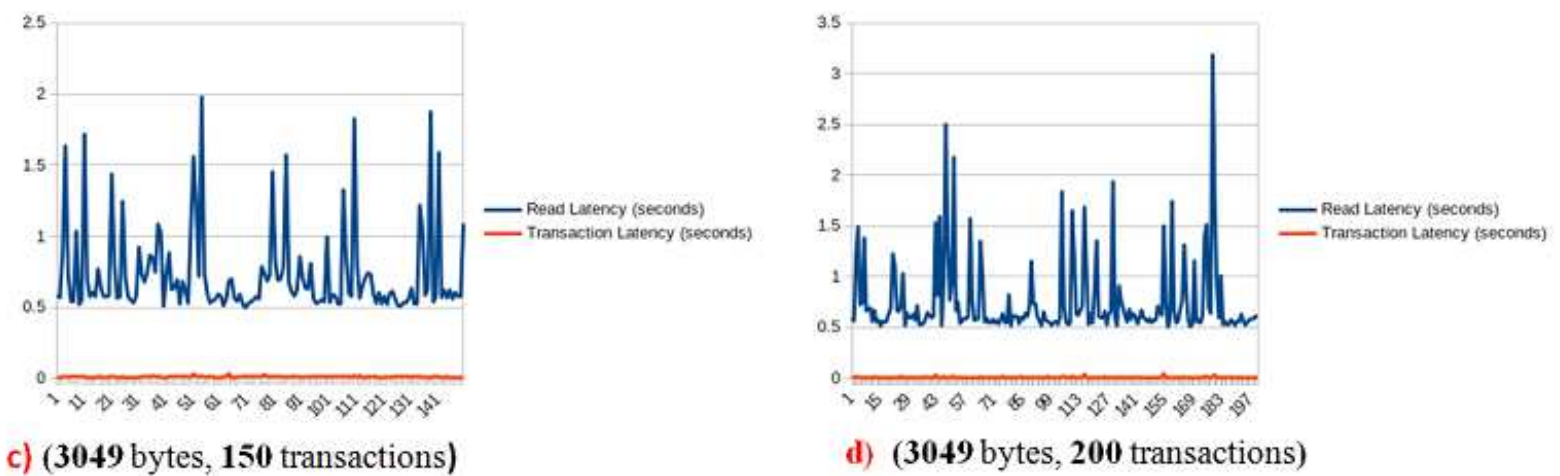

d) (3049 bytes, 200 transactions)
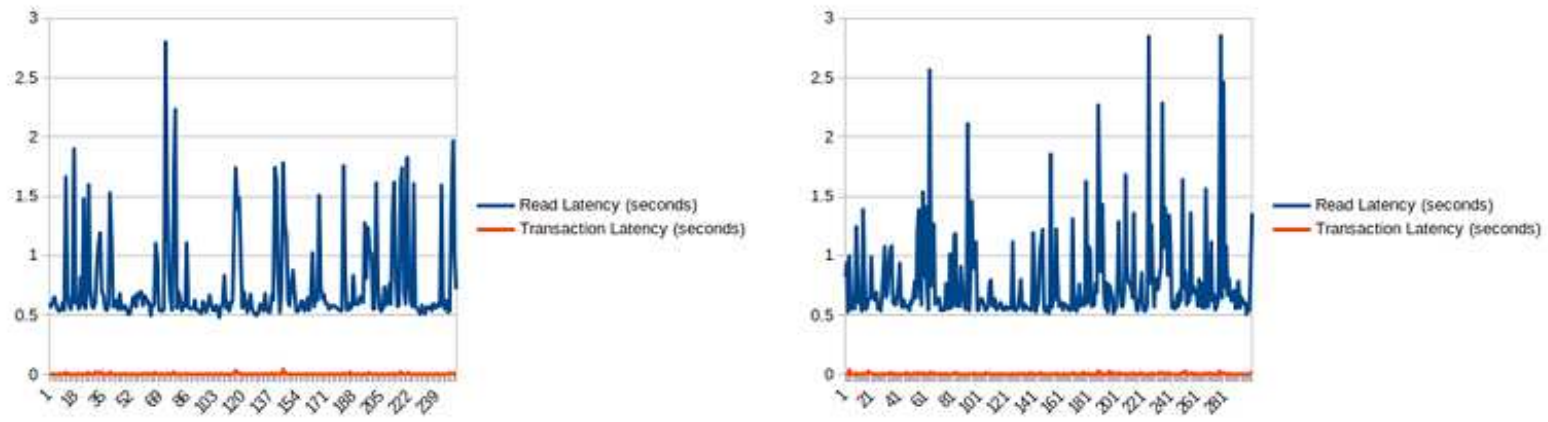

e) (3049 bytes, 250 transactions)

f) (3049 bytes, 300 transactions)

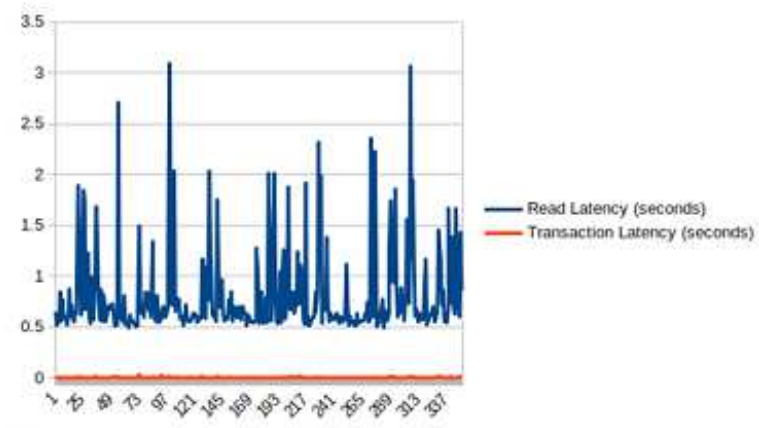

g) (3049 bytes, $\mathbf{3 5 0}$ transactions)

Figure 6. impact of a transaction data size of 3049 bytes on RL and TL for 50, 100,150,200,250,300, and 350 transactions. 


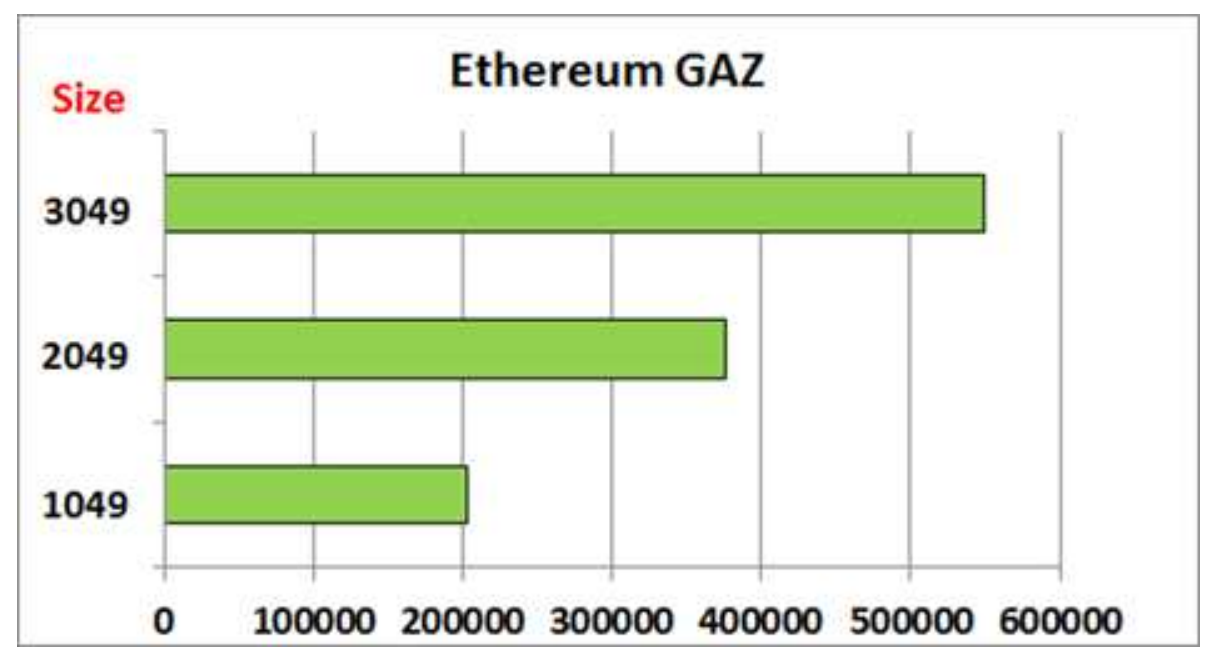

Figure 7. Ethereum GAZ consumption of satellite transactions with three data sizes.

Tables 4-6 illustrate the impacts of the three transaction data sizes (i.e., 1049, 2049, and 3049 bytes) and GAS on RT and TT. Figures 8-10 depict the behavior of RT and TT with changes in the number of satellite transactions

\begin{tabular}{|l|l|l|l|l|}
\hline Satellite Trans. & Read Throughput(RPS) & Transaction Throughput(TPS) & Gas & Size(bytes) \\
\hline $\mathbf{5 0}$ & 112.95 & 2.95 & 202419 & 1049 \\
\hline $\mathbf{1 0 0}$ & 68.19 & 1.92 & 202419 & 1049 \\
\hline $\mathbf{1 5 0}$ & 50.90 & 1.48 & 202419 & 1049 \\
\hline $\mathbf{2 0 0}$ & 41.41 & 1.20 & 202419 & 1049 \\
\hline $\mathbf{2 5 0}$ & 33.87 & 0.99 & 202419 & 1049 \\
\hline $\mathbf{3 0 0}$ & 28.32 & 0.83 & 202419 & 1049 \\
\hline $\mathbf{3 5 0}$ & 26.15 & 0.76 & 202419 & 1049 \\
\hline
\end{tabular}

Table 4. Results of the impact of a transaction data size of 1049 bytes on RT, and TT according to the number of satellites transactions.

\begin{tabular}{|l|l|l|l|l|}
\hline Satellite Trans. & Read Throughput(RPS) & Transaction Throughput(TPS) & Gas & Size(bytes) \\
\hline $\mathbf{5 0}$ & 112.7 & 2.35 & 375750 & 2049 \\
\hline $\mathbf{1 0 0}$ & 71.92 & 1.19 & 375750 & 2049 \\
\hline $\mathbf{1 5 0}$ & 51.46 & 1.05 & 375750 & 2049 \\
\hline $\mathbf{2 0 0}$ & 42.21 & 0.67 & 375750 & 2049 \\
\hline $\mathbf{2 5 0}$ & 34.29 & 0.49 & 375750 & 2049 \\
\hline $\mathbf{3 0 0}$ & 30.9 & 0.39 & 375750 & 2049 \\
\hline $\mathbf{3 5 0}$ & 17.14 & 0.28 & 375750 & 2049 \\
\hline
\end{tabular}

Table 5. Results of the impact of a transactional data size of 2049 bytes on RT, and TT according to the number of satellites transactions. 


\begin{tabular}{|l|l|l|l|l|}
\hline Satellite Trans. & Read Throughput(RPS) & Transaction Throughput(TPS) & Gas & Size(bytes) \\
\hline $\mathbf{5 0}$ & 112.61 & 1.53 & 549084 & 3049 \\
\hline $\mathbf{1 0 0}$ & 76.39 & 1.01 & 549084 & 3049 \\
\hline $\mathbf{1 5 0}$ & 55.71 & 0.72 & 549084 & 3049 \\
\hline $\mathbf{2 0 0}$ & 43.81 & 0.56 & 549084 & 3049 \\
\hline $\mathbf{2 5 0}$ & 36.98 & 0.46 & 549084 & 3049 \\
\hline $\mathbf{3 0 0}$ & 31.61 & 0.38 & 549084 & 3049 \\
\hline $\mathbf{3 5 0}$ & 27.80 & 0.33 & 549084 & 3049 \\
\hline
\end{tabular}

Table 6. Results of the impact of a transaction data size of 3049 bytes on RT, and TT according to the number of satellites transactions

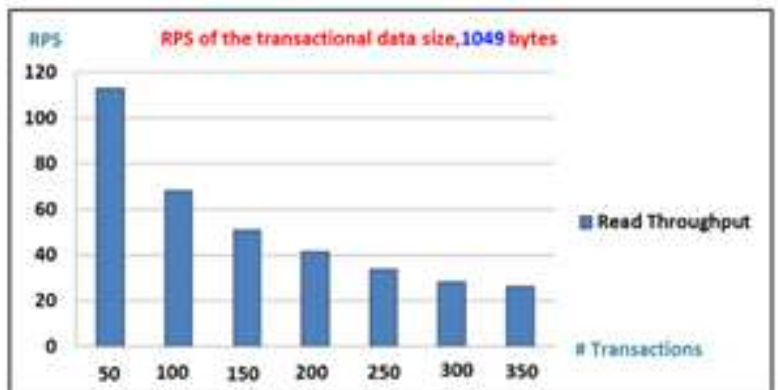

a)

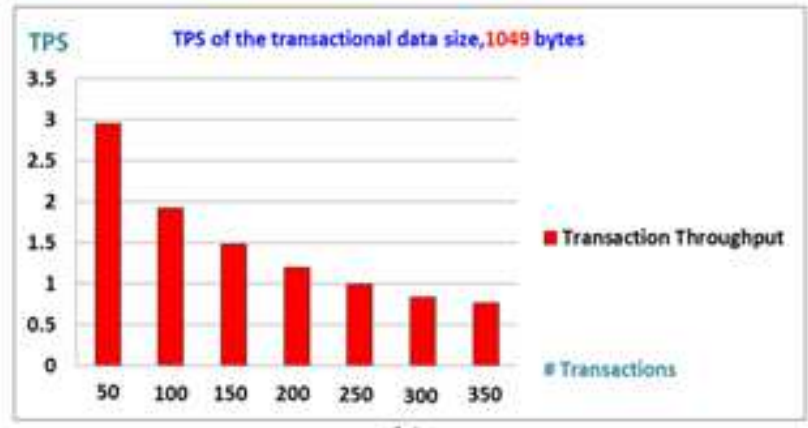

b)

Figure 8. a) Read throughput and b) Transaction Throughput of satellite transactional data size, 1049 bytes.

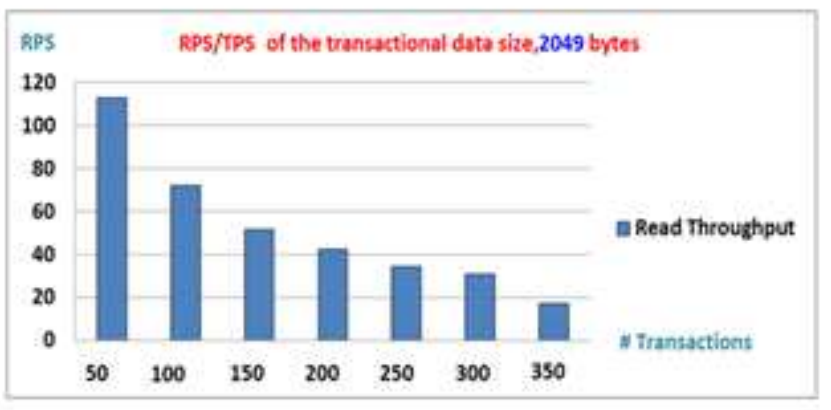

a)

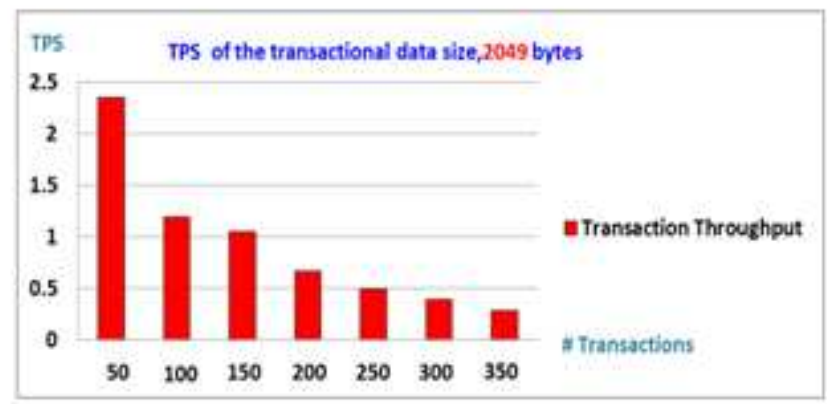

b)

Figure 9. a) Read throughput and b) transaction throughput for a satellite transaction data size of 2049 bytes. 


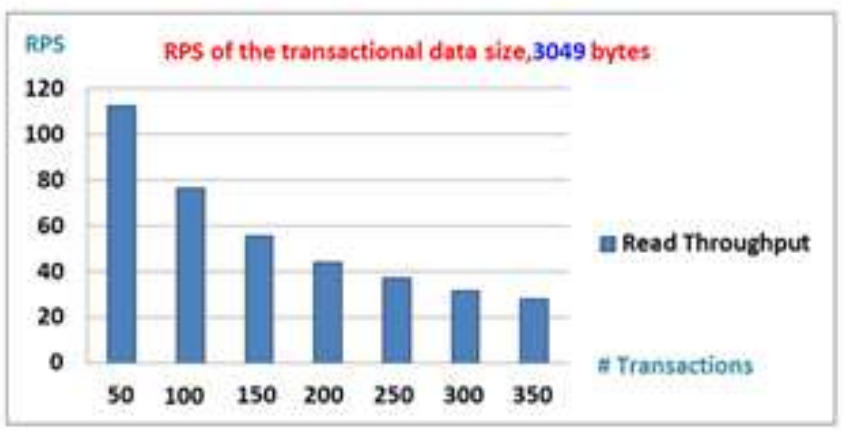

a)

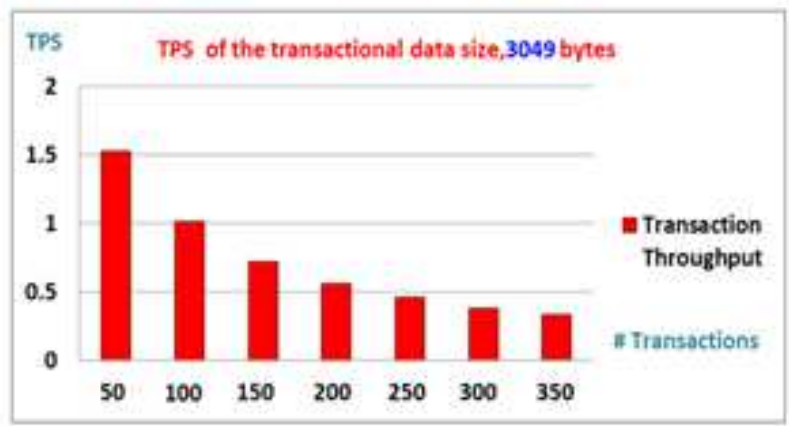

b)

Figure 10. a) Read throughput and b) transaction throughput for a satellite transaction data size, of 3049 bytes.

\subsubsection{PoST Authenticity and Reliability Results}

The next investigation assesses the authenticity and reliability of PoST in processing satellite transactions. We reconfigured the dataset to consist of trusted transactions and fake transactions within an individual swarm and in two swarms. Then, we applied the PoST protocol to the reconfigured dataset to test the efficiency of the proposed protocol in accepting trusted satellite transactions and rejecting fake transactions. This ability can be measured by creating a confusion matrix of, true positives (TPs), true negatives (TNs), false positives (FPs), and false negatives (FNs) and then calculating true positive rate (TPR), true negative rate (TNR), and accuracy as shown in table 7

$$
\text { TruePositiveRate }(\text { TPR })=T P /(\text { Postivecases }(P))
$$

$$
\text { TrueNegativeRate }(\text { TNR })=T N /(\text { Negativecases }(N))
$$

$$
\text { Accuracy }=(T P+T N) /(P+N)
$$

\begin{tabular}{|l|l|l|l|l|l|}
\hline Transactions. & TP & TN & TPR & TNR & Accuracy \\
\hline $\mathbf{1 0 0}$ & 50 & 50 & $100 \%$ & $100 \%$ & $100 \%$ \\
\hline $\mathbf{2 0 0}$ & 100 & 100 & $100 \%$ & $100 \%$ & $100 \%$ \\
\hline $\mathbf{3 0 0}$ & 150 & 150 & $100 \%$ & $100 \%$ & $100 \%$ \\
\hline
\end{tabular}

Table 7. TPR, TNR, and accuracy of the PoST protocol for verifying satellite transactions

\section{Discussion}

Studies that have noted the importance of using blockchain technology in the space industry are still few and immature. However, some reports have shown that blockchain will be a base technology in developing the next generation of open-source satellites and spacecraft ${ }^{31},{ }^{32}$. Some research efforts discussed the adoption of blockchain to solve various challenges in the space industry and satellite communications. These challenges include the security and privacy of satellite communications ${ }^{27,28}$ and satellite data broadcasting ${ }^{29}$.

An initial objective of this study was to propose the concept of SDTs to build a new blockchain protocol called PoST. The first question in this study sought to determine the efficiency of PoST in managing satellite transactions within a swarm of satellites. The answer to this question included various interesting results. First, the impact of the number of satellite transitions (where the size of a transaction is 1049 bytes) on the transaction latency of blockchain when PoST is applied ( as shown in Figure 4) is not significant, as it did not increase the transaction latency by more than 0.05 TPS, although the read latency has a variable relationship with the number of satellite transactions. Therefore, satellite transactions can be quickly processed, 
even with an increasing number of satellite transactions and increasing variance of the read latency. Moreover, Figure 8 shows that the impact of the number of transactions on the total RT and TT of blockchain has a reverse relationship. This finding demonstrates that the proposed PoST protocol can quickly process transactions within a swarm of satellites regardless of the number of transactions, confirming the efficiency of PoST in managing satellite transactions within a single swarm.

The second question in this study sought to determine the efficiency of PoST in managing satellite transactions between different swarms of satellites. In answering this question, it was found that the number of satellite transactions does not have a substantial impact (when the transaction size is 2049 or 3049 bytes) on the TL of blockchain when PoST is applied, as shown in Figures 5 and 6. These results confirm that an increasing number of satellite transactions exchanged between satellites in different swarms does not increase the TL by more than 0.05 TPS. In addition, Figures 9 and 10 illustrate that the impact of the number of transactions on the total RT and TT of blockchain has a reverse relationship. Thus, the proposed PoST protocol is efficient in managing satellite transactions between different swarms of satellites, regardless of the number of transactions.

Another important finding is the impact of transaction data size on RT and TT. The results indicated that the transactional data size $(1049,2049$, and 3049 bytes) has a reverse relationship with RT and TT, which confirms the efficiency of PoST in processing transaction data between satellites, as depicted in Figures 8, 9, and 10

The third question in this research was to investigate the reliability of the PoST protocol in verifying valid transactions within trusted swarms of satellites. As shown in Table 7, the authentication process reaches $100 \%$ in terms of the accuracy, true positive rate (TPR), and true negative rate (TNR) of the PoST protocol. These results hold whether the satellites are in the same swarm or different swarms.

These findings broadly support the work in ${ }^{27}$ regarding the use of blockchain to verify satellite access. Although the results are in line with those in $^{27}$, PoST has some advantages that make it better than the blockchain-based access verification protocol (BAVP) proposed $\mathrm{in}^{27}$ :

1. PoST has been tested against the key measures of blockchain (RL, TL, RT, and TT), while BAVP has not. This makes the results of this study the leading ones.

2. Although the BAVP achieved good results regarding response time and delay of satellite transactions, it does not consider either the size of the transaction data or the frequency of transactions, as in the PoST protocol. Thus, the results of the PoST protocol may be more dependable than those of BAVP

3. In BAVP, the test scenarios focused on only the computation time of the encryption algorithm, (identity-based encryption (IBE)) used in BAVP compared with RSA without introducing any results about blockchain impacts on the efficiency of BAVP, as shown in the PoST protocol.

4. In BAVP, the authors claimed that the protocol has intrinsic resistance to replay attacks, man-in -the-middle attacks, impersonation attacks, and denial-of-service attacks, but there is no evaluation confirming this claim. In contrast, the reliability of PoST against fake satellite transactions has been tested, and good results have been obtained (see Table 7).

Further research efforts should be undertaken to investigate the effectiveness of the proposed PoST protocol for managing and authenticating two other types of transactions in two case studies:

1. Investigate how PoST can manage and authenticate tracking and data relay satellite (TDRS) transactions to increase the amount of communication time between satellites and ground stations and to improve the amount and speed of uplink and downlink data that could be transferred to or from the TDRS system.

2. Investigate how PoST can be used to immunize a satellite constellation against space debris collisions based on the sensing orbital data between satellites and space debris. Managing and securing this type of transaction using the proposed PoST is another important research problem.

\section{Conclusion}

This study aimed to introduce a new blockchain protocol called proof of space transactions (PoST) to model, manage and authenticate satellite transactions within various constellations (or swarms). The main contribution of this study showed that tokenizing space transactions in the form of space digital tokens (SDTs) and processing them using the proposed PoST protocol is a promising solution for managing and authenticating space transactions based on blockchain technology. This study has demonstrated the effectiveness of the PoST protocol based on simulation experiments that proved the effectiveness of the proposed blockchain protocol in managing and authenticating satellite transactions in P2P networks. According to the results, this study is among the first mature attempts to thoroughly investigate tokenizing space transactions and processing them using blockchain technology. Therefore, these results are expected to improve space communications management to develop a new 
generation of blockchain-based satellite systems. This study could be further enhanced by conducting additional experimental simulations and evaluations to investigate the effectiveness of the proposed PoST protocol for studying two important problems in this study: first, managing and authenticating TDRS satellites and ground station transactions.; second, immunizing satellite constellations against space debris collisions based on the sensing orbital data between satellites and space debris.

\section{References}

1. Team, S. F. E. Global space economy grows in 2019 to $\$ 423.8$ billion, the space report $2020 \mathrm{q} 2$ analysis shows. Space Foundation https://www.spacefoundation.org/2020/07/30/ global-space-economy-grows-in-2019-to-423-8-billion-the-space-report-2020-q2-analysis-shows/\#: :text=Global\% 20Space\%20Economy\%20Grows\%20in, Q2\%20Analysis\%20Shows\%20\%2D\%20Space\%20Foundation (2020).

2. Pelton, J. N., Madry, S. \& Camacho-Lara, S. Satellite applications handbook: The complete guide to satellite communications, remote sensing, navigation, and meteorology. Handb. Satell. Appl. New York: Springer 3-17 (2013).

3. Martin, A.-S. \& Freeland, S. The advent of artificial intelligence in space activities: New legal challenges. Space Policy 55, 101408 (2021).

4. Nanjangud, A., Blacker, P. C., Bandyopadhyay, S. \& Gao, Y. Robotics and ai-enabled on-orbit operations with future generation of small satellites. Proc. IEEE 106, 429-439 (2018).

5. Luis, J. J. G. et al. Artificial intelligence algorithms for power allocation in high throughput satellites: A comparison. In 2020 IEEE Aerospace Conference, 1-15 (IEEE, 2020).

6. Fraire, J. A., Céspedes, S. \& Accettura, N. Direct-to-satellite iot-a survey of the state of the art and future research perspectives. In International Conference on Ad-Hoc Networks and Wireless, 241-258 (Springer, 2019).

7. Routray, S. K. et al. Satellite based iot for mission critical applications. In 2019 International Conference on Data Science and Communication (IconDSC), 1-6 (IEEE, 2019).

8. Chien, W.-C., Lai, C.-F., Hossain, M. S. \& Muhammad, G. Heterogeneous space and terrestrial integrated networks for iot: Architecture and challenges. IEEE Netw. 33, 15-21 (2019).

9. Zhang, Z., Zhang, W. \& Tseng, F.-H. Satellite mobile edge computing: Improving qos of high-speed satellite-terrestrial networks using edge computing techniques. IEEE network 33, 70-76 (2019).

10. Wang, Y., Yang, J., Guo, X. \& Qu, Z. A game-theoretic approach to computation offloading in satellite edge computing. IEEE Access 8, 12510-12520 (2019).

11. Denby, B. \& Lucia, B. Orbital edge computing: Nanosatellite constellations as a new class of computer system. In Proceedings of the Twenty-Fifth International Conference on Architectural Support for Programming Languages and Operating Systems, 939-954 (2020).

12. Bacsardi, L. On the way to quantum-based satellite communication. IEEE Commun. Mag. 51, 50-55 (2013).

13. Bedington, R., Arrazola, J. M. \& Ling, A. Progress in satellite quantum key distribution. npj Quantum Inf. 3, 1-13 (2017).

14. Gibney, E. Chinese satellite is one giant step for the quantum internet. Nat. News 535, 478 (2016).

15. Liao, S.-K. et al. Satellite-to-ground quantum key distribution. Nature 549, $43-47$ (2017).

16. Feng, M. \& Xu, H. Msnet-blockchain: A new framework for securing mobile satellite communication network. In 2019 16th Annual IEEE International Conference on Sensing, Communication, and Networking (SECON), 1-9 (IEEE, 2019).

17. Li, M., Wang, L. \& Zhang, Y. A framework for rocket and satellite launch information management systems based on blockchain technology. Enterp. Inf. Syst. 1-15 (2019).

18. Surdi, S. A. Space situational awareness through blockchain technology. J. Space Saf. Eng. 7, 295-301 (2020).

19. Blockstream. The bitcoin blockchain from space. no internet required. Blockstream https://blockstream.com/satellite/ (2021).

20. Wainscott-Sargent, A. Blockchain: The next big disruptor in space. satellitetoday.com http://interactive.satellitetoday.com/ blockchain-the-next-big-disruptor-in-space/ (2021).

21. Chaturvedi, A. Singapore startup to build world's first open-source satellite network. geospatialworld.net https://www. geospatialworld.net/blogs/singapore-startup-to-build-worlds-first-open-source-satellite-network/ (2021).

22. Ulybyshev, Y. Satellite constellation design for complex coverage. J. Spacecr. Rocket. 45, 843-849 (2008).

23. Alshaer, M. K. Cyber attacks on satellites review \& solutions. . 
24. G, J. \& I, R. Hacking a bird in the sky: Hijacking very small aperture terminal (vsat) connections:. geospatialworld.net https://conference.hitb.org/hitbsecconf2006kl/materials/DAY\%202\%20-\%20Jim\%20Geovedi\%20and\% 20Raditya\%20Iryandi\%20-\%20Hacking\%20a\%20bird\%20in\%20the\%20sky.pdf (2021).

25. Wu, Z., Zhang, Y., Yang, Y., Liang, C. \& Liu, R. Spoofing and anti-spoofing technologies of global navigation satellite system: A survey. IEEE Access 8, 165444-165496 (2020).

26. Clark, L., Tung, Y.-C., Clark, M. \& Zapanta, L. A blockchain-based reputation system for small satellite relay networks. In 2020 IEEE Aerospace Conference, 1-8 (IEEE, 2020).

27. Wei, S., Li, S., Liu, P. \& Liu, M. Bavp: blockchain-based access verification protocol in leo constellation using ibe keys. Secur. Commun. Networks 2018 (2018).

28. Xu, R., Chen, Y., Blasch, E. \& Chen, G. Exploration of blockchain-enabled decentralized capability-based access control strategy for space situation awareness. Opt. Eng. 58, 041609 (2019).

29. Zhang, Y.-H. \& Liu, X. F. Satellite broadcasting enabled blockchain protocol: A preliminary study. In 2020 Information Communication Technologies Conference (ICTC), 118-124 (IEEE, 2020).

30. Zhao-wei, S., Hong, D., Wei-chao, Z. \& Xian-de, W. Attacking satellite path planning based on genetic algorithm. $J$. Aerosp. Eng. 25, 32-38 (2012).

31. Jones, K. L. Blockchain in the space sector. center for space policy and strategy https://aerospace.org/sites/default/files/ 2020-03/Jones_Blockchain_03052020.pdf (2020).

32. Spacechain.com. decentralized space agency. Spacechain.com https://spacechain.com/wp-content/uploads/2018/09/ SpaceChain-White-Paper.pdf (2021).

\section{Author contributions statement}

M.T proposed the idea, M.T, A.E.H, and T.G. suggested the methodology, M.T and T.G. conceived the experiments, E.G. conducted the experiments, M.T, A.E.H, and T.G. analysed the results, M.T and T.G. and E.G, wrote the original draft, M.T, A.E.H, V.S., and T.G. reviewed and edited the final version. All authors have read and agreed to the published version of the manuscript. 